\title{
Frequency of Play and Exercise at Preschool and Childhood Ages: Effect on Exercise Habits and Physical Fitness in Adolescence
}

\author{
Takayoshi Yamada $^{1, *}$, Shinichi Demura ${ }^{2}$, Shigeru Shimada ${ }^{3}$ \\ ${ }^{1}$ Faculty of Education, Humanities and Social Sciences, University of Fukui, Fukui, Japan \\ ${ }^{2}$ Institute of Human and Social Sciences, Kanazawa University, Kanazawa, Japan \\ ${ }^{3}$ Faculty of Sports and Health Sciences, Fukui University of Technology, Fukui, Japan \\ *Corresponding author: yamadat@u-fukui.ac.jp
}

Received January 04, 2019; Revised February 08, 2019; Accepted February 18, 2019

\begin{abstract}
This study aimed to clarify the effect of play frequency and exercise when at preschool and childhood ages on the exercise habits and physical fitness of those who are currently adolescents. Healthy young men $(\mathrm{N}=600)$ from 15 to 21 years old (age: $16.5 \pm 1.2$ years, height: $171.1 \pm 5.7 \mathrm{~cm}$, body mass: $61.0 \pm 10.0 \mathrm{~kg}$ ) participated in the physical fitness test of the Ministry of Education, Culture, Sports, Science and Technology (MEXT) after answering questions about current exercise habits and frequency of play and exercise at their preschool and childhood ages. Significant relationships among frequency of play at preschool age and exercise in childhood and current exercise habits (Cramer's $\mathrm{V}=0.10-0.15, \mathrm{p}<0.028$ ) were observed. Although no significant difference was found in the physique among the four groups, which differed in the frequency of play and exercise in early childhood and childhood, performance on the 50-m dash, standing long jump, handball throw, and 20-m shuttle run were significantly superior in groups with higher frequency of play and exercise. The present results may indicate that frequency of play at preschool age and exercise in childhood significantly affects subsequent exercise habits and physical fitness levels.
\end{abstract}

Keywords: play, exercise, preschool, childhood, exercise habit, physical fitness

Cite This Article: Takayoshi Yamada, Shinichi Demura, and Shigeru Shimada, "Frequency of Play and Exercise at Preschool and Childhood Ages: Effect on Exercise Habits and Physical Fitness in Adolescence." Journal of Physical Activity Research, vol. 4, no. 1 (2019): 62-66. doi: 10.12691/jpar-4-1-8.

\section{Introduction}

Children today may be deprived of outdoor environments where they can play. They often spend a considerable amount of time in relatively motionless play, for instance, watching television and playing electronic games (Ministry of Health, Labour and Welfare; Health Japan 21). It has been proven that physical activity in adults has reduced rates of mortality, not only from cardiovascular disease and all causes of mortality, but also from risk of cancer, type 2 diabetes mellitus, and depression [1,2,3,4,5].

Meanwhile, evidence regarding the morbidity and mortality of adults who hardly exercised as children is very scarce, but morbidity and mortality in adulthood may decrease if childhood exercise habits are favorable [6]. Recently, Pälve et al. examined long-term effects of physical activity on carotid artery elasticity after 21 years of follow up with children and young adults [7]. They reported that physical activity in boys and young adults was associated with carotid artery elasticity in later life. Thus, higher levels of physical activity in youth may benefit future cardiovascular health. As a result, evidence supporting the notion that childhood physical activity relates to future health status has been emerging.

Others have also reported that current physical fitness relates to present exercise habits [8], but it also relates, to a lesser degree, to past exercise habits [9]. In other words, even if people have had good exercise habits in the past, if they are inactive after that, physical fitness decreases, and the risk of lifestyle-related diseases increases. Meanwhile, preschool and lower grades of elementary school are a critical period (pre-golden age) for learning fundamental movements. Presumably, developing fundamental movements until reaching the nearly mature stage in the above-mentioned critical period contributes to smooth development of technical motor skills in childhood, adolescence, and adulthood [10]. Moreover, Suzuki and Nishijima reported that current exercise habits moderately relate to past exercise habits [9]. Namely, the frequency of active play in early childhood and exercise during childhood will affect subsequent exercise and sports habits. Therefore, although it is assumed that frequency of play and exercise before and during childhood contributes to maintaining and enhancing physical fitness in adolescence or adulthood, there is little evidence supporting this assumption. 
This study aimed to clarify the effect of frequency of active play in early childhood and exercise during childhood on exercise habits and physical fitness in adolescence.

\section{Methods}

\subsection{Subjects}

Subjects were 600 healthy adolescent male students, aged 15-21 $(16.5 \pm 1.2)$ years, belonging to the National College of Technology. Height and weight were $171.1 \pm$ $5.7 \mathrm{~cm}$ and $61.0 \pm 10.0 \mathrm{~kg}$, respectively, and their physical characteristics were comparable with Japanese standard values. Each subject provided informed consent following the full explanation of the experiment to them, and the experimental protocol was approved by the Ethics Committee on Human Experimentation, Faculty of Education, Kanazawa University (authorization number: 19-4).

\subsection{Measurement items}

Each subject's physical fitness was evaluated based on the eight items (grip strength, sit ups, trunk flexion, repeated sideways jump, 20-m shuttle run, 50-m dash, standing long jump, and handball throw) of a fitness test developed by the MEXT in Japan. Each physical fitness item was measured on the MEXT (Department of Sports and Youth of the MEXT [11]) implementation guideline. Current exercise habits were evaluated by the following items: sports club membership (belonging or not belonging), exercise frequency (almost every day (more than 3 days/week), sometimes (1-2 days/week), occasionally (1-2 days/month), or never), and exercise amount per day (less than $30 \mathrm{~min}, 30-60 \mathrm{~min}, 60-120 \mathrm{~min}$, or over $120 \mathrm{~min})$.

Subjects' frequency of play at preschool age was evaluated by how frequently they had performed active, vigorous physical activities outdoors before entering elementary school (never, occasionally, or frequently). Frequency of exercise in childhood was evaluated by whether the boys frequently performed some exercise and played sports (never, 1-2 days/month, 1-2 days/week, 3-4 days/week, or more than 5 days/week). In addition, physical fitness tests and surveys were conducted. Health and physical education faculty with expert knowledge and experience conducted physical fitness tests during regular classes once a week.

\subsection{Grouping Based on Frequency of Play at Preschool Age and Exercise in Childhood}

Subjects who responded "never" or "occasionally" to the question, "Did you play actively and vigorously during outdoor physical activity before entering elementary school?” formed a lower play-frequency group (L); those who answered "frequently" formed a higher play-frequency group (H). Subjects who responded "never" or "1-2 days/month" to "Did you play sports and exercise during higher elementary school grades?” formed a lower exercise-frequency group (L); those who answered "1-2 days/week," "3-4 days/week," or "more than 5 days/week" formed a higher exercise-frequency group $(\mathrm{H})$. Based on this information, the following four groups were formed: an LL group with low frequency of play at both preschool age and exercise in childhood: $(\mathrm{n}=65$, age $=16.5 \pm 1.2$ years, height $=169.7 \pm 5.7 \mathrm{~cm}$, body mass $=59.0 \pm 9.3 \mathrm{~kg}$ ); an $\mathrm{LH}$ group with lower frequency of play at preschool age, but higher frequency of exercise in childhood: $(n=135$, age $=16.3 \pm 1.2$ years, height $=171.6 \pm 5.9 \mathrm{~cm}$, body mass = $61.3 \pm 9.3 \mathrm{~kg}$ ); an HL group with higher frequency of play at preschool age, but lower frequency of exercise in childhood: $(n=81$, age $=16.8 \pm 1.1$ years, height $=171.1 \pm 5.5 \mathrm{~cm}$, body mass $=$ $61.8 \pm 9.8 \mathrm{~kg}$ ); and an $\mathrm{HH}$ group with high frequency of play at both preschool age and exercise in childhood: $(\mathrm{n}=$ 319 , age $=16.4 \pm 1.2$ years, height $=171.2 \pm 5.6 \mathrm{~cm}$, body mass $=61.1 \pm 10.5 \mathrm{~kg})$.

\subsection{Statistical Analysis}

Cramer's V was calculated to examine relationships of frequency of play at preschool age and exercise in childhood, with factors such as belonging to a sports club, exercise frequency, and exercise amount per day. Mean differences in age, physique, and physical fitness test scores among these four groups were examined using oneway analysis of variance. Tukey's honest significant difference was conducted for post hoc analysis. Statistical significance was at a probability level of $<0.05$.

\section{Results}

Table 1 -Table 3 show associations of frequency of play at preschool age and exercise in childhood with the following factors: currently belonging or not belonging to a sports club, exercise frequency, and exercise amount per day. All associations were significant (Cramer's $V=0.10-0.15$, $\mathrm{p}<0.028$ ). Table 4 shows results of independent one-way analysis of variance and multiple comparisons for age and physique among the four groups of $\mathrm{HH}, \mathrm{LH}, \mathrm{HL}$, and LL (see "Methods") based on frequency of play at preschool age and exercise in childhood. No significant differences were found among groups for any item. Table 5 shows results of independent one-way analysis of variance and multiple comparisons for physical fitness tests among groups based on frequency of play at preschool age and exercise in childhood. Significant differences were found in the 20-m shuttle run, 50-m dash, standing long jump, handball throw, repeated sideways jump, and physical fitness score. These performances in the $\mathrm{HH}$ group (high frequency of play and exercise) were superior to those in the LL group (low frequency of play and exercise).

Table 1. The association of frequency of play in preschool children age and exercise in childhood with or without belonging to sports clubs

\begin{tabular}{cccccc}
\hline \multicolumn{5}{c}{ With or without belonging to sports clubs } \\
& Belonging & Not Belonging & Total & V & p \\
\cline { 1 - 4 } LL & 33 & 32 & 65 & & \\
LH & 48 & 87 & 135 & & \\
HL & 47 & 34 & 81 & 0.15 & 0.003 \\
HH & 125 & 194 & 319 & & \\
\cline { 1 - 3 } Total & 253 & 347 & 600 & & \\
\hline
\end{tabular}


Table 2. The association of frequency of play in preschool children age and exercise in childhood with the current exercise frequency

\begin{tabular}{|c|c|c|c|c|c|c|c|}
\hline & \multicolumn{7}{|c|}{ Current exercise frequency } \\
\hline & Almost everyday & Sometimes & Occasionally & Never & Total & $\mathrm{V}$ & $\mathrm{p}$ \\
\hline LL & 16 & 13 & 18 & 18 & 65 & & \\
\hline LH & 58 & 38 & 20 & 19 & 135 & & \\
\hline HL & 25 & 24 & 19 & 13 & 81 & 0.10 & 0.028 \\
\hline $\mathrm{HH}$ & 134 & 78 & 61 & 46 & 319 & & \\
\hline Total & 233 & 153 & 118 & 96 & 600 & & \\
\hline
\end{tabular}

Table 3. The association of frequency of play in preschool children age and exercise in childhood with the current exercise time per day

\begin{tabular}{|c|c|c|c|c|c|c|c|}
\hline & \multicolumn{7}{|c|}{ Current exercise time per day } \\
\hline & Less than $30 \mathrm{~min}$ & $30-60 \mathrm{~min}$ & $60-120 \min$ & Over 120 min & Total & V & $\mathrm{P}$ \\
\hline LL & 33 & 8 & 20 & 4 & 65 & & \\
\hline $\mathrm{LH}$ & 32 & 23 & 38 & 42 & 135 & & \\
\hline HL & 33 & 5 & 11 & 22 & 81 & 0.13 & $<0.001$ \\
\hline $\mathrm{HH}$ & 93 & 47 & 85 & 94 & 319 & & \\
\hline Total & 190 & 92 & 153 & 162 & 600 & & \\
\hline
\end{tabular}

Table 4. Mean and standard deviations for age and physique among 4 groups of HH, LH, HL, and LL based on frequency of play in a preschool children age and exercise in the childhood

\begin{tabular}{|c|c|c|c|c|c|c|}
\hline & & $\mathrm{n}$ & Mean & SD & $\mathrm{F}$ & $\mathrm{p}$ \\
\hline \multirow{4}{*}{ Age } & LL & 65 & 16.5 & 1.2 & \multirow{4}{*}{2.36} & \multirow{4}{*}{0.071} \\
\hline & $\mathrm{LH}$ & 135 & 16.3 & 1.2 & & \\
\hline & $\mathrm{HL}$ & 81 & 16.8 & 1.1 & & \\
\hline & $\mathrm{HH}$ & 319 & 16.4 & 1.2 & & \\
\hline \multirow{4}{*}{ Height } & LL & 65 & 169.7 & 5.7 & \multirow{4}{*}{1.58} & \multirow{4}{*}{0.193} \\
\hline & $\mathrm{LH}$ & 135 & 171.6 & 6.0 & & \\
\hline & $\mathrm{HL}$ & 81 & 171.1 & 5.6 & & \\
\hline & $\mathrm{HH}$ & 319 & 171.2 & 5.6 & & \\
\hline \multirow{4}{*}{ Body mass } & LL & 65 & 59.0 & 9.4 & \multirow{4}{*}{1.11} & \multirow{4}{*}{0.346} \\
\hline & $\mathrm{LH}$ & 135 & 61.3 & 9.4 & & \\
\hline & HL & 81 & 61.8 & 9.8 & & \\
\hline & $\mathrm{HH}$ & 319 & 61.1 & 10.5 & & \\
\hline \multirow{4}{*}{$\%$ body fat } & LL & 65 & 12.8 & 5.2 & \multirow{4}{*}{1.11} & \multirow{4}{*}{0.346} \\
\hline & $\mathrm{LH}$ & 135 & 14.4 & 6.7 & & \\
\hline & HL & 81 & 14.2 & 6.1 & & \\
\hline & $\mathrm{HH}$ & 319 & 13.6 & 5.7 & & \\
\hline
\end{tabular}

Table 5. Mean and standard deviation for physical fitness tests among 4 groups based on frequency of play in a preschool children age and exercise in the childhood

\begin{tabular}{|c|c|c|c|c|c|c|c|}
\hline & & $\mathrm{n}$ & Mean & SD & $\mathrm{F}$ & $\mathrm{P}$ & Post hoc \\
\hline \multirow{4}{*}{ 50-meter dash } & LL & 62 & 8.0 & 0.8 & \multirow{4}{*}{3.37} & \multirow{4}{*}{0.018} & \multirow{4}{*}{$\mathrm{LL}>\mathrm{HH}, \mathrm{HL}$} \\
\hline & LH & 134 & 7.8 & 0.5 & & & \\
\hline & HL & 80 & 7.7 & 0.4 & & & \\
\hline & $\mathrm{HH}$ & 315 & 7.7 & 0.5 & & & \\
\hline \multirow{4}{*}{ Standing long jump } & LL & 64 & 212.6 & 24.6 & \multirow{4}{*}{6.59} & \multirow{4}{*}{$<0.001$} & \multirow{4}{*}{$\begin{array}{c}\text { HL, HH > LL } \\
\text { HL > LH }\end{array}$} \\
\hline & LH & 135 & 218.5 & 21.4 & & & \\
\hline & HL & 80 & 226.6 & 20.2 & & & \\
\hline & $\mathrm{HH}$ & 318 & 223.0 & 21.1 & & & \\
\hline \multirow{4}{*}{ Handball throw } & LL & 63 & 21.6 & 5.2 & \multirow{4}{*}{6.03} & \multirow{4}{*}{$<0.001$} & \multirow{4}{*}{$\mathrm{HH}, \mathrm{LH}, \mathrm{HL}>\mathrm{LL}$} \\
\hline & LH & 134 & 24.1 & 5.0 & & & \\
\hline & HL & 81 & 24.0 & 4.5 & & & \\
\hline & $\mathrm{HH}$ & 313 & 24.4 & 4.7 & & & \\
\hline \multirow{4}{*}{ 20-meter shuttle run } & LL & 61 & 72.9 & 16.6 & \multirow{4}{*}{5.64} & \multirow{4}{*}{$<0.001$} & \multirow{4}{*}{$\mathrm{HH}, \mathrm{LH}>\mathrm{LL}$} \\
\hline & LH & 135 & 82.1 & 19.4 & & & \\
\hline & HL & 78 & 78.6 & 16.2 & & & \\
\hline & $\mathrm{HH}$ & 314 & 83.4 & 20.5 & & & \\
\hline \multirow{4}{*}{ Repeated sideways jump } & LL & 63 & 56.0 & 3.9 & \multirow{4}{*}{2.89} & \multirow{4}{*}{0.035} & \multirow{4}{*}{$\mathrm{HH}>\mathrm{LL}$} \\
\hline & LH & 134 & 57.0 & 5.9 & & & \\
\hline & HL & 79 & 57.2 & 4.9 & & & \\
\hline & $\mathrm{HH}$ & 316 & 57.9 & 5.1 & & & \\
\hline
\end{tabular}




\begin{tabular}{|c|c|c|c|c|c|c|c|}
\hline & & $\mathrm{n}$ & Mean & SD & $\mathrm{F}$ & $\mathrm{P}$ & Post hoc \\
\hline \multirow{4}{*}{ Grip strength } & LL & 64 & 41.4 & 7.4 & \multirow{4}{*}{2.12} & \multirow{4}{*}{0.097} & \\
\hline & LH & 134 & 42.8 & 7.1 & & & \\
\hline & HL & 81 & 44.2 & 6.4 & & & \\
\hline & $\mathrm{HH}$ & 315 & 42.8 & 6.4 & & & \\
\hline \multirow{3}{*}{ Sit-ups } & LL & 62 & 28.2 & 5.1 & \multirow{3}{*}{2.08} & \multirow{3}{*}{0.102} & \\
\hline & $\mathrm{LH}$ & 135 & 29.1 & 5.1 & & & \\
\hline & $\mathrm{HH}$ & 315 & 29.8 & 5.4 & & & \\
\hline \multirow{4}{*}{ Trunk anteflexion } & LL & 63 & 48.7 & 9.6 & \multirow{4}{*}{0.07} & \multirow{4}{*}{0.977} & \\
\hline & $\mathrm{LH}$ & 134 & 48.9 & 8.9 & & & \\
\hline & HL & 81 & 48.5 & 9.4 & & & \\
\hline & $\mathrm{HH}$ & 316 & 49.0 & 9.7 & & & \\
\hline \multirow{3}{*}{ Total score } & $\mathrm{LL}$ & 65 & 45.9 & 11.3 & \multirow{3}{*}{6.78} & \multirow{3}{*}{$<0.001$} & \multirow{3}{*}{$\mathrm{HH}, \mathrm{HL}, \mathrm{LH}>\mathrm{LL}$} \\
\hline & HL & 81 & 50.8 & 8.4 & & & \\
\hline & $\mathrm{HH}$ & 319 & 51.5 & 8.9 & & & \\
\hline
\end{tabular}

\section{Discussion}

Results show a significant relationship between frequency of play at preschool age and exercise in childhood with present exercise habits (Table 1, Table 2, and Table 3). Clearly, the amount of physical activity at preschool age and during childhood relates to subsequent exercise habits. Suzuki and Nishijima measured eight physical fitness items of MEXT and 61 items regarding achievement of movement to clarify causal relationships among physical fitness, exercise habits, and exercise experience [9]. They reported that experience in exercise and sports affects physical fitness and exercise skill, but experience affects the acquisition of exercise habits even more. Exercise habits largely affect physical fitness more than exercise skill, and sports experience affects exercise skill more than physical fitness. In other words, the above findings suggest that past exercise and sports experience greatly affect subsequent exercise habits. The present results also suggest that the frequency of play at preschool age and exercise in childhood relate to exercise habits in adolescence, which is in alignment with Suzuki and Nishijima's report [9]. However, characteristics of subjects' physiques were similar among the four groups, regardless of the frequency of play at preschool age and exercise in childhood. Beginning in 1986, Pälve et al. measured ultrasound-derived indices of carotid artery elasticity in addition to investigating physical activity available during leisure time [7]. In 2007, Pälve's group studied 1417 children and 999 young adults to clarify long-term effects of physical activity on carotid artery elasticity after 21 years of follow up. They reported that physical activity related closely to carotid artery elasticity as compared with the general indices of lifestyle diseases such as physique, blood pressure, low-density lipoprotein and high-density lipoprotein cholesterol, triglycerides, insulin, and blood glucose. Additionally, higher level of physical activity in children may benefit their future cardiovascular health. The present study differs from Pälve et al.’s study [7] because cross-sectional data are used, and outcomes reflecting health status are not evaluated. Therefore, the likelihood of contracting disease cannot be predicted based on present results. However, a relationship between physique before childhood and exercise habits was not observed in either this study or in Pälve et al.'s study [7]. Exercise frequency before and during childhood affects exercise habits in adolescence, as mentioned. From these facts, there is a high possibility that exercise frequency before and during childhood affects subsequent health status.

Meanwhile, frequency of play at preschool age and exercise in childhood reveals significant relationships to current physical fitness levels. Individuals with higher frequency of play and exercise have higher physical fitness levels (Table 5). Suzuki and Nishijima examined the following criteria of exercise and sports for improvement of physical fitness and motor ability in youth: exercise for three days or more per week and two hours or more per day, sports experience of two or more events before entering high school, and commencement of sports before graduating from elementary school [8]. Most of all, the latter two findings may suggest that frequency of play at preschool age and exercise in childhood affects subsequent physical fitness. Indeed, the preschool and childhood periods are especially suitable for developing fundamental motor ability, because the nervous system develops markedly during those periods. In this study, significant differences among groups with different exercise frequencies are also found in the following sport activities: 50-m dash, standing long jump, and handball throw, which represent running, jumping, and throwing. This suggests that good exercise habits before and during childhood affect the development of motor ability in adolescence. However, such differences are not found in grip strength, sit ups, and trunk flexion. Malina reported that although physical activity shows a low-to-moderate degree of transfer from adolescence to adulthood, inactivity transfers to a high degree [12]. Meanwhile, reports suggest that although motor ability and healthrelated fitness (strength, flexibility, sports skill, and aerobic power) are significantly transferred, the amount of transfer is low to moderate. The present results also show similarity with Malina's report [12], which revealed carryover effects of exercise frequency and subsequent physical fitness. However, it was inferred that among physical fitness elements, some do not carry over. Meanwhile, the total score of physical fitness in persons with a high frequency of play and exercise is superior to that in 
persons with a low frequency of activity in the preschool and childhood periods. From the above information, it is considered that frequency of play and exercise in preschool age and childhood years affects subsequent exercise habits and physical fitness levels.

\section{Conclusion}

Frequency of play and exercise in preschool age and childhood years may affect subsequent exercise habits and physical fitness levels.

\section{References}

[1] Blair, S.N., Kampert, J.B., Kohl, H.W 3rd., Barlow, C.E., Macera, C.A., Paffenbarger, R.S Jr., Gibbons, L.W., "Influences of cardiorespiratory fitness and other precursors on cardiovascular disease and all-cause mortality in men and women," Journal of American Medical Association, 276 (3). 205-210. Jul. 1996.

[2] Lee, I.M., Sesso, H.D., Oguma, Y., Paffenbarger, R.S Jr., "Relative intensity of physical activity and risk of coronary heart disease," Circulation, 107 (8). 1110-1116. Mar. 2003a.

[3] Lee, I.M., Sesso, H.D., Oguma, Y., Paffenbarger, R.S Jr., "Physical activity, body weight, and pancreatic cancer mortality," British Journal of Cancer, 88 (5). 679-683. Mar. 2003b.

[4] Sato, Y., Nagasaki, M., Nakai, N., Fushimi, T., "Physical exercise improves glucose metabolism in lifestyle-related diseases,"
Experimental Biology and Medicine (Maywood), 228 (10). 12081212. Nov. 2003.

[5] Feskanich, D., Willett, W., Colditz, G., "Walking and leisure-time activity and risk of hip fracture in postmenopausal women," Journal of American Medical Association, 288 (18). 2300-2306. Nov. 2002.

[6] Baba, R., "What will become of the child who does not exercise (Special issue: Exercise bring up child)," Journal of Health, Physical Education and Recreation, 58. 305-310. 2008.

[7] Pälve, K.S., Pahkala, K., Magnussen, C.G., Koivistoinen, T., Juonala, M., Kähönen, M., Lehtimäki, T., Rönnemaa, T., Viikari, J.S., Raitakari, O.T., "Association of physical activity in childhood and early adulthood with carotid artery elasticity 21 years later: the cardiovascular risk in Young Finns Study,” Journal of American Heart Association, 3 (2). e000594. Apr. 2014.

[8] Suzuki, K., Nishijima, T., "Criteria of exercise and sports for improvement of physical fi tness and motor ability in youth (15-18 year olds)," Human Performance Measurement, 3. 11-20. 2006.

[9] Suzuki, K., Nishijima, T., "Effects of sports experience and exercise habits on physical fitness and motor ability in high school students," School Health, 1. 22-38. 2005.

[10] Kobayashi, K., Sugihara, T., Torii, S., Noda, H., Ishiduka, H., "Sports in youth period," Certified Sports Leader Education Text, Japan Sports Association, 123-144. 2005.

[11] Ministry of Education, Culture, Sports, Science and Technology., "Implementation guideline of a new physical fitness test (For 6579 years old),"

<http://www.mext.go.jp/component/a_menu/sports/detail/_icsFil es/afieldfile/2010/07/30/1295079_04.pdf>.

[12] Malina, R.M., "Tracking of physical activity and physical fitness across the lifespan,” Research Quarterly for Exercise and Sport, 67 (3 Suppl). S48-S57. Sep.1996. 